\title{
CALCULATION OF EXCITONIC ABSORPTION SPECTRUM OF GaAs QUANTUM WIRE FREE-STANDING IN VACUUM
}

\author{
P. VAGNER ${ }^{a}$, D. MUNZAR ${ }^{b}$ AND M. MošKo $a$ \\ ${ }^{a}$ Institute of Electrical Engineering, Slovak Academy of Sciences \\ Dúbravská cesta 9, 84239 Bratislava, Slovak Republic \\ ${ }^{b}$ Department of Solid State Physics, Faculty of Science \\ Masaryk University, Kotlářská 2, 611 37, Czech Republic
}

The electron-hole interaction in a thin $(\approx 10 \mathrm{~nm}) \mathrm{GaAs}$ quantum wire free-standing in vacuum is strongly enhanced by the image charge due to the abrupt permittivity drop at the wire surface. As a result, the exciton binding energies are much larger and the exciton wave functions much more localised than those of the GaAs quantum wire surrounded by the AlGaAs. The absorption spectrum of the free-standing wire shows besides the $1 \mathrm{~s}$ exciton peak also the $3 s$ and $5 s$ exciton peaks, even if the peaks are $15 \mathrm{meV}$ broad. The continuum absorption edge shows a large blue shift due to the renormalization of single-particle energies by the image charge.

PACS numbers: 73.20.-r, 73.20.Dx

The free-standing quantum wells and wires are thin films, bars, or whiskers supported only on their ends or connected to the substrate only on the side with the smallest cross-section [1]. In thin $(\approx 10 \mathrm{~nm})$ structures the electron-hole $(\mathrm{e}-\mathrm{h})$ Coulomb interaction is enhanced by the image charge due to the small permittivity of the surrounding medium (vacuum or air). We have shown [2] that in a thin free-standing GaAs quantum well the enhanced $c$-h interaction enhances the exciton binding energy by almost one order of magnitude compared to the AlGaAs/GaAs/AlGaAs quantum well. As a result the absorption spectrum is strongly modified: the $2 s$ and $3 s$ exciton peaks are distinguished in addition to the $1 s$ peak, and the continuum absorption edge shows a large blue shift due to the renormalization of single-particle energies by the image charge. In free-standing wires these effects can be expected to be stronger, as supported by recent calculations of exciton binding energies [3]. In this work we'verify these expectations by calculating the absorption spectrum of a cylindrical $\mathrm{GaAs}$ quantum wire free-standing in vacuum.

We start with the derivation of the $\mathrm{e}-\mathrm{h}$ interaction energy. We consider an undoped infinitely long wire with a cylindrical cross-section in the $y-z$ plane and with the wire axis associated with the $x$-axis. At the wire surface there is an abrupt 
permittivity drop from $\epsilon_{s}=12.5 \epsilon_{0}$ to $\epsilon_{0}$, where $\epsilon_{s}$ and $\epsilon_{0}$ are the permittivities of GaAs and vacuum, respectively. Assuming at the wire surface an infinitely high potential barrier, the envelope function of the carrier ground state is given in the effective mass approximation as $\psi_{11}(r)=J_{0}\left(x_{01} \frac{r}{R}\right) /\left[\sqrt{\pi} R J_{1}\left(x_{01}\right)\right]$, where $J_{0}, J_{1}$ are the Bessel functions, $x_{01}$ is the first zero of the $J_{0}, r=\sqrt{y^{2}+z^{2}}$, and $R$ is the wire radius. The corresponding carrier charge density is $\rho(r)=e\left|\psi_{11}(r)\right|^{2}$. The potential $\varphi\left(x-x_{\mathrm{h}}, r\right)$ created at point $x, r$ inside the wire by that charge density (say by a hole positioned at $x=x_{\mathrm{h}}$ ) obeys the Poisson equation

$$
\nabla\left[\epsilon(r) \nabla \varphi\left(x-x_{\mathrm{h}}, r\right)\right]=-\rho(r) \delta\left(x-x_{\mathrm{h}}\right),
$$

where $\epsilon(r)=\epsilon_{0}+\left(\epsilon_{s}-\epsilon_{0}\right) \theta(R-r)$ and $\theta(R-r)$ is the IIeaviside step function. The Poisson equation in cylindrical coordinates reads

$$
\begin{aligned}
& \left(\frac{\partial^{2}}{\partial x^{2}}+\frac{\partial^{2}}{\partial r^{2}}+\frac{1}{r} \frac{\partial}{\partial r}\right) \varphi\left(x-x_{\mathrm{h}}, r\right)=-\frac{\rho(r)}{\epsilon_{s}} \delta\left(x-x_{\mathrm{h}}\right)-\frac{\rho_{\mathrm{im}}\left(x-x_{\mathrm{h}}, r\right)}{\epsilon_{s}}, \\
& \rho_{\mathrm{im}}\left(x-x_{\mathrm{h}}, r\right)=\left(\frac{\epsilon_{s}}{\epsilon(r)}-1\right) \rho(r) \delta\left(x-x_{\mathrm{h}}\right)+\frac{\epsilon_{s}}{\epsilon(r)} \frac{\partial \epsilon(r)}{\partial r} \frac{\partial \varphi\left(x-x_{\mathrm{h}}, r\right)}{\partial r},
\end{aligned}
$$

where $\rho_{\mathrm{im}}\left(x-x_{\mathrm{h}}, r\right)$ is the image charge density. The Fourier transform of (1) gives

$$
\left(\frac{\partial^{2}}{\partial r^{2}}+\frac{1}{r} \frac{\partial}{\partial r}-Q_{x}^{2}\right) \varphi\left(Q_{x}, r\right)=-\frac{\rho(r)}{\epsilon_{s}}-\frac{\rho_{\mathrm{im}}\left(Q_{x}, r\right)}{\epsilon_{s}},
$$

where

$$
\varphi\left(Q_{x}, r\right)=\int_{-\infty}^{\infty} \mathrm{d}\left(x-x_{\mathrm{h}}\right) \mathrm{e}^{\mathrm{i} Q_{x}\left(x-x_{\mathrm{h}}\right)} \varphi\left(x-x_{\mathrm{h}}, r\right)
$$

and

$$
\rho_{\text {im }}\left(Q_{x}, r\right)=\int_{-\infty}^{\infty} \mathrm{d}\left(x-x_{\mathrm{h}}\right) \mathrm{e}^{\mathrm{i} Q_{x}\left(x-x_{\mathrm{h}}\right) \rho_{\mathrm{im}}}\left(x-x_{\mathrm{h}}, r\right) .
$$

The solution of (3) with the right hand side replaced by $\delta\left(r-r_{0}\right)$ is the Green function $G\left(Q_{x}, r, r_{0}\right)=-I_{0}\left(Q_{x} r^{<}\right) K_{0}\left(Q_{x} r^{>}\right)$, where $I_{0}$ and $K_{0}$ are the modified Bessel functions, $r^{<}=\min \left(r, r_{0}\right)$, and $r^{>}=\max \left(r, r_{0}\right)$. The general solution of (3),

$$
\varphi\left(Q_{x}, r\right)=\int_{0}^{\infty} \mathrm{d} r_{0} r_{0} G\left(Q_{x}, r, r_{0}\right)\left[-\rho\left(r_{0}\right)-\rho_{\mathrm{im}}\left(Q_{x}, r_{0}\right)\right] / \epsilon_{s},
$$

allows us [4] to derive

$$
\varphi\left(Q_{x}, r\right)=-\frac{e}{\epsilon_{s}}\left[G_{11}\left(Q_{x}, r\right)+\frac{\epsilon_{s}-\epsilon_{0}}{\epsilon_{0}} \frac{R G\left(Q_{x}, r, R\right) \frac{\partial G_{11}\left(Q_{x}, r=R\right)}{\partial r}}{1-\frac{\epsilon_{x}-\epsilon_{0}}{\epsilon_{0}} R \frac{\partial G\left(Q_{x}, r=R, R\right)}{\partial r}}\right],
$$

where $G_{11}\left(Q_{x}, r\right)=\int_{0}^{R} \mathrm{~d} r_{0} r_{v} G\left(Q_{x}, r, r_{0}\right)\left|\psi_{11}\left(r_{0}\right)\right|^{2}$. Finally, the effective $\mathrm{e}-\mathrm{h}$ interaction

$$
-e \varphi_{\mathrm{e}-\mathrm{h}}^{\mathrm{eff}}\left(x_{\mathrm{e}}-x_{\mathrm{h}}\right)=-e \int_{-\infty}^{\infty} \mathrm{d} Q_{x} \mathrm{e}^{-\mathrm{i} Q_{x}\left(x_{\mathrm{e}}-x_{\mathrm{h}}\right)} \varphi_{11}\left(Q_{x}\right),
$$

is the inverse Fourier transform of the function $\varphi_{11}\left(Q_{x}\right)=\int_{0}^{R} \mathrm{~d} r \quad r \varphi\left(Q_{x}, r\right)$ $\times\left|\psi_{11}(r)\right|^{2}$. The second term on the right hand side of (4) is the potential due to 
the image charge $\rho_{\mathrm{im}}$. It disappears when $\epsilon_{0} \rightarrow \epsilon_{s}$, i.e., when the wire is buried in the AlGaAs (we ignore a small difference between the permittivity of GaAs and AlGaAs).

When the wave function of the e-h pair, $\Psi\left(x_{e}-x_{h}, r_{e}, r_{h}\right)$, is approximated as $\psi_{11}\left(r_{\mathrm{e}}\right) \psi_{11}\left(r_{\mathrm{h}}\right) \Phi\left(x_{\mathrm{e}}-x_{\mathrm{h}}\right)$, the eigenenergy of the e-h pair can be expressed as

$$
E_{\mathrm{g}}=E_{0}+\frac{\hbar^{2}}{2}\left(\frac{1}{m_{\mathrm{e}}}+\frac{1}{m_{\mathrm{h}}}\right)\left(\frac{x_{01}}{R}\right)^{2}+\sigma_{\mathrm{e}}+\sigma_{\mathrm{h}}-E_{\mathrm{b}},
$$

where $E_{\mathrm{b}}$ and $\Phi(x)$ satisfy the equation

$$
\left[-\frac{\hbar^{2}}{2}\left(\frac{1}{m_{\mathrm{e}}}+\frac{1}{m_{\mathrm{h}}}\right) \frac{\partial^{2}}{\partial x^{2}}-e \varphi_{\mathrm{e}-\mathrm{h}}^{\mathrm{eff}}(x)\right] \Phi(x)=-E_{\mathrm{b}} \Phi(x) .
$$

Here $E_{0}$ is the energy gap of bulk GaAs, $\frac{\hbar^{2}}{2}\left(\frac{1}{m_{\mathrm{e}}}+\frac{1}{m_{\mathrm{h}}}\right)\left(x_{01} / R\right)^{2}$ is the carrier confinement energy, $\sigma_{\mathrm{e}}$ and $\sigma_{\mathrm{h}}$ are the electron and hole selfenergies (Eq. (9)), and $E_{\mathrm{b}}$ is the exciton binding energy. The electron and hole effective masses, $m_{\mathrm{e}}$ and $m_{\mathrm{h}}$, are taken as $0.067 m_{0}$ and $0.2 m_{0}$. In the two-band model the choice $m_{\mathrm{h}}=0.2 m_{0}$ fits the dispersion of the ground hole subband resulting from the heavy and light hole mixing [5].
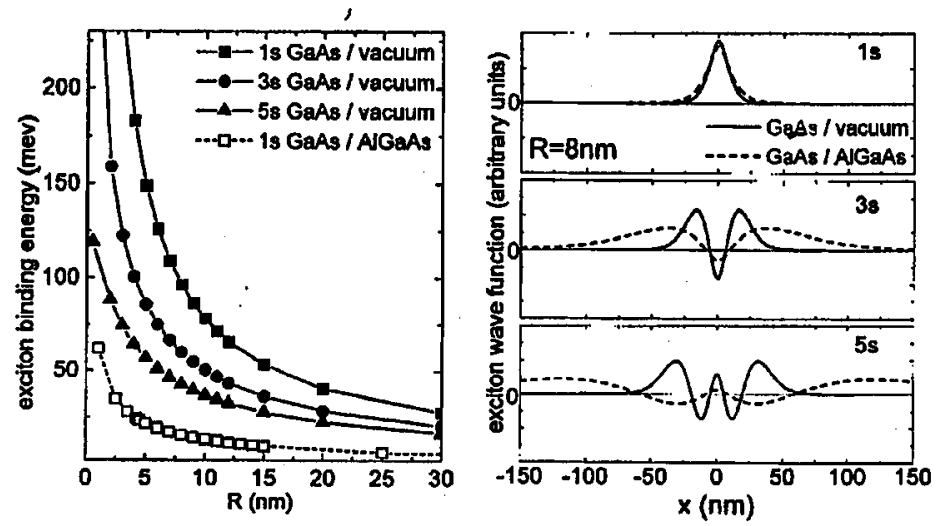

Fig. 1. The exciton binding energy versus the wire radius $R$ and the exciton wave function for $R=8 \mathrm{~nm}$. Three lowest states with even wave functions (labelled $1 s, 3 s, 5 s$ ) are shown. The solid lines are the results for the free-standing $\mathrm{GaAs}$ wire, the dashed lines are the results for the GaAs wire buried in the AlGaAs.

Figure 1 shows the exciton binding energy versus the wire radius $R$ and the exciton wave function $\Phi(x)$ for $R=8 \mathrm{~nm}$. The solid lines are the results for the free-standing GaAs wire, the dashed lines are the results for the GaAs wire buried in the AlGaAs. Only the three lowest states with even wave functions (labelled $1 s, 3 s, 5 s)$ are shown, since mainly these states contribute to the excitonic absorption. In the free-standing wire the binding energies are much larger and the wave functions are more localized. This is due to the enhanced $e-h$ interaction (see Fig. 2). Figure 2 shows the effective e-h interaction energy $-e \phi_{\mathrm{e}-\mathrm{h}}^{\mathrm{eff}}$ versus the $\mathrm{e}-\mathrm{h}$ 
distance $x$ for the wire radius $R=8 \mathrm{~nm}$. The thick solid line is obtained for the free-standing wire, the thick dashed line for the buried wire. In the former case the $\mathrm{e}-\mathrm{h}$ interaction is much stronger, which is due to the image charge effect (the second term on the right hand side of (4)). In the latter case the effect disappears.

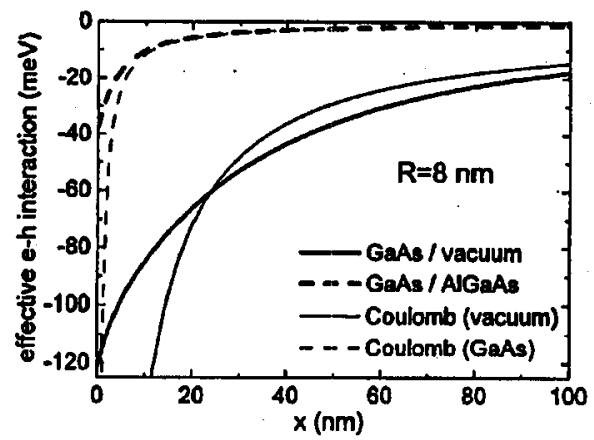

Fig. 2. The effective $e-h$ interaction energy versus the e-h distance for the wire radius $R=8 \mathrm{~nm}$. The thick solid line is the result for the free-standing GaAs wire, the thick dashed line for the buried GaAs wire. Also shown is the Coulomb e-h energy of two point-like charges in vacuum (thin solid line) and in GaAs (thin dashed line).

The absorption coefficient of the quantum wire versus the photon energy $(E)$ reads [6]

$$
\alpha=\frac{\xi_{1}}{E} \frac{\theta\left(E-E_{\mathrm{g}}^{\prime}\right)}{\sqrt{E-E_{\mathrm{g}}^{\prime}}}+\frac{\xi_{2}}{E} \sum_{i=1}^{\infty} \delta\left(E-E_{\mathrm{g}}^{\prime}+E_{\mathrm{b}, i}\right)\left|\Phi_{i}(x=0)\right|^{2},
$$

where $E_{\mathrm{g}}^{\prime}$ is the energy of the continuum edge (the right hand side of (6) without $\left.E_{\mathrm{b}}\right)$, the sum extends over all the bound exciton states,

$$
\begin{aligned}
& \xi_{1}=\left(P^{2} e^{2} \sqrt{2 \mu}\right) /\left(2 \epsilon_{0} c n_{r} m_{0}^{2} \pi R^{2}\right), \\
& \xi_{2}=\left(P^{2} e^{2} \pi \hbar\right) /\left(\epsilon_{0} c n_{r} m_{0}^{2} \pi R^{2}\right),
\end{aligned}
$$

$P$ is the matrix element between the valence band $p$ state and the conduction band $s$ state at $\Gamma$ for $\operatorname{GaAs}\left(P^{2} / m_{0}=11.3 \mathrm{eV}\right), n_{r}=3.7$ is the refraction index of $\mathrm{GaAs}$, and $\mu=m_{\mathrm{e}} m_{\mathrm{h}} /\left(m_{\mathrm{e}}+m_{\mathrm{h}}\right)$. The first and second terms in (8) describe the continuum and the exciton contribution, respectively. Since the first term is derived [6] in the single electron approximation, it should be multiplied by the Sommer Peld factor. Here we use the Sommerfeld factor values taken from Fig. 2b of Ref. [7]. Since these values were obtained for a buried GaAs wire, they likely overestimate the Sommerfeld factor of the free-standing wire. We also incorporate the inhomogeneous broadening by convoluting the spectrum (8) with a Gaussian function $\exp \left[-\left(E_{\mathrm{g}}^{\prime}-\left\langle E_{\mathrm{g}}^{\prime}\right\rangle\right)^{2} /\left(2 \Delta{E_{\mathrm{g}}^{\prime}}^{2}\right)\right]$, where $\left\langle E_{\mathrm{g}}^{\prime}\right\rangle$ and $\Delta E_{\mathrm{g}}^{\prime}$ are the mean value and the mean deviation of $E_{\mathrm{g}}^{\prime}$.

In Fig. 3 we compare the absorption spectra of the free-standing wire and buried wire. The broadening $2 \Delta E_{\mathrm{g}}^{\prime}=10 \mathrm{meV}$ is considered here. The distance between the $1 s$ exciton peak and the onset of the continuum is the $1 s$ exciton 


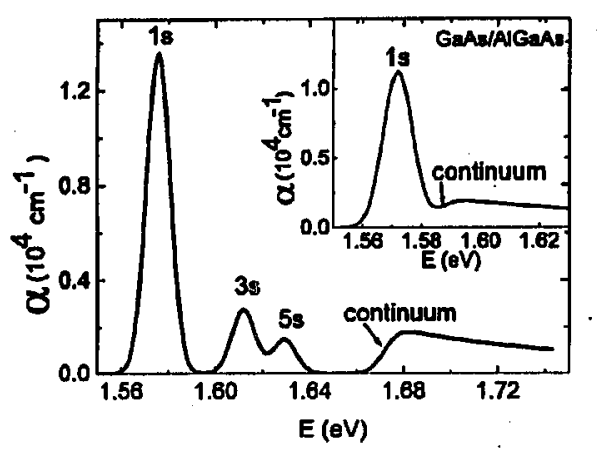

Fig. 3. Absorption coefficient of the free-standing GaAs quantum wire with $8 \mathrm{~nm}$ radius. The inset shows the spectrum of the buried GaAs quantum wire.

binding energy. It is 6.5 times larger for the free-standing wire than for the buried wire. For the former case also the $3 s$ and $5 s$ peaks are visible. The $5 s$ peak tends to smear out when $2 \Delta E_{\mathrm{g}}^{\prime}>15 \mathrm{meV}$. Another remarkable feature is the $84 \mathrm{meV}$ blue shift of the free-standing wire spectrum. The blue shift is determined by the $\sigma_{\mathrm{e}}+\sigma_{\mathrm{h}}$. The selfenergy $\sigma_{\gamma}, \gamma=\mathrm{e}, \mathrm{h}$, is the increment of the carrier energy due to the image charge. It is given as

$$
\sigma_{\gamma}=2 \pi \int_{0}^{R} \mathrm{~d} r_{\gamma} r_{\gamma} \sigma_{\gamma}\left(r_{\gamma}\right)\left|\psi_{11}\left(r_{\gamma}\right)\right|^{2}
$$

where $\sigma_{\gamma}\left(r_{\gamma}\right)=(e / 2) \int_{-\infty}^{\infty} \mathrm{d} Q_{x} \int_{0}^{R} \mathrm{~d} r r\left|\psi_{11}(r)\right|^{2}$ (second term on the right hand side of (4)). ciated.

Support from the BankPro Team a.s. and from the agency VEGA is appre-

\section{References}

[1] M.D. Williams, S.C. Shunk, M.G. Young, D.P. Docter, D.M. Tennant, B.I. Miller, Appl. Phys. Lett. 61, 1353 (1992); K. Hiruma, M. Yazawa, K. Haraguchi, K. Ogawa, T. Katsuyama, M. Koguchi, H. Kakibayashi, J. Appl. Phys. 74, 3162 (1993).

[2] M. Moško, D. Munzar, P. Vagner, Phys. Rev. $B$, in press.

[3] J.B. Xia, K.W. Cheah, Phys. Rev. B 55, 1596 (1997).

[4] P. Vagner, D. Munzar, M. Moško, to be published.

[5] R. Cingolani, R. Rinaldi, M. Ferrara, Phys. Rev. B 48, 14331 (1993).

[6] G. Bastard, Wave Mechanics Applied to Semiconductor Heterostructures, Halsted Press, Les Ulis 1988.

[7] T. Ogawa, T. Takagahara, Phys. Rev. B 44, 8138 (1991). 